\title{
Evaluation of Esthetics and Marginal Adaptation of Composite Restorations for Fractured Permanent Incisors
}

\author{
Ibrahim H El-kalla ${ }^{1}$, Hanaa Mahmoud Shalan ${ }^{2 *}$ and Mai M Mahmoud ${ }^{3}$ \\ ${ }^{1}$ Professor of Pediatric Dentistry, Faculty of Dentistry, Mansoura University, Egypt \\ ${ }^{2}$ Associate Professor of Pediatric Dentistry, Faculty of Dentistry, Mansoura University, Egypt \\ ${ }^{3} B D S$ faculty of Dentistry, Mansoura University, Egyptt \\ *Corresponding Author: Hanaa Mahmoud Shalan, Associate Professor of Pediatric Dentistry, Faculty of Dentistry, Mansoura University, \\ Egypt. E-mail: shalanhm93@gmail.com
}

Received: October 09, 2019; Published: October 30, 2019

DOI: $10.31080 /$ ASDS.2019.03.0683

\begin{abstract}
Background: Fractured incisors is a problem in pediatric dentistry that affect children Aim: Evaluation of esthetics and marginal adaptation of fractured incisors restored with composite resins.

Design: Sixty central incisors were selected, embedded in plastic molds filled with dental stone. Standardized mesio-incisal angle fracture was performed. Teeth were divided into 2 equal groups. Group I: Filtek Z350XT and group II: Ceram X duo. Each group was subdivided equally into 3 subgroups. (A): no mechanical preparation, (B): circumferential shoulder with bevel, (C): circumferential chamfer at fracture line. Teeth were restored with composite resin, thermocycled and photographed. Esthetic evaluation was performed by Photoshop computer programme using CIE Lab system. Teeth were sectioned to evaluate marginal adaptation using stereomicroscope and scanning electron microscope.

Results: No significant color difference was observed between subgroups in group I and group II. Statistical analysis of marginal adaptation data revealed good adaptation with no significant difference between subgroups in group I Filtek Z350XT and between subgroups in group II Ceram X duo. There was no significant difference between subgroups A, B and C in both groups.

Conclusion: Both type of composite and all 3 types of preparations provides acceptable color match and esthetic restorations with well adapted margin.

Keywords: Fractured Incisor; Composite; Preparation; Marginal Adaptation; Color Match
\end{abstract}

\section{Introduction}

Traumatic Dental injuries (TDIs) are a challenge to dentists all over the world. It is often seen in children before school, at school, and young adults mainly due to fall, contact sports, and car accidents. Uncomplicated crown fracture is the most frequent results of traumatic dental injury in the permanent dentition. Maxillary central incisors are the mostly affected teeth. TDIs to anterior teeth affect the patient both esthetically and psychologically. The most occurring traumatic injuries are crown fracture, which represents $92 \%$ of traumatic injuries of permanent teeth [1-5].

Fractured anterior teeth affects the patient functionally, esthetically, and may cause psychological distress to the patient $[6,7]$. So, dealing with fractured teeth should be done urgently to maintain their vitality, regain esthetic appearance, and function $[8,9]$. 
Incisal edges fracture can be restored with different treatment modalities to regain their original shape and color as reattachment of fractured part if the fractured part was available $[8,10]$, If not found, composite restoration is the main choice for restoring fractured teeth [11]. Re-attachment of fractured tooth is considered excellent biologic approach, having the advantage of providing, low cost, conservative approach, no need for laboratory procedures, and psychological benefits [12]. Direct composite resin restorations of fractured anterior teeth with enamel and dentine fracture has the advantages of minimal removal of tooth structure, material biocompatibility, low cost, and shortening chair time [13].

Various conservative designs of enamel preparation for enhancing retention and esthetic of composite resin have been evolved over time taking the advances in material and adhesive technology within considerations [14].

Different studies were performed to evaluate the effect of fractured margin preparation on the longevity and esthetic appearance of direct adhesive restoration. Some researchers believed that, no need for fracture margin preparation to improve either esthetics nor function of direct composite restoration [15-18]. Others preferred bevel at fractured margin to increase retention and gave better esthetic as it provided gradual color transition from composite to tooth [19-21].

Some researchers recommended chamfer preparation as it provided well defined margin so that the restoration can be finished adequately, with adequate marginal integrity, and good retention [22]. Some authors recommended shoulder with bevel as it provides esthetic appearance by masking fracture line and conservative approach [23].

Evaluation of esthetic restoration in term of color match is done either visually or by devices as (spectrophotometer, colorimeter, and image analysis technique). Color measurement by devices is usually performed by CIELab system [24]. The esthetic and durability of the anterior teeth restoration depends on the quality of marginal adaptation so adequate marginal integrity increase the longevity of the restoration [25].The esthetic and marginal adaptation test of biomaterials and techniques is a pre-requisite for their safe and effective clinical use; so it fruitful to test two different enamel preparation designs in anterior permanent teeth using two type of composite resin materials.

\section{Materials and Methods}

Teeth selection: Sixty caries-free human permanent maxillary central incisors extracted for periodontal reasons were selected. Teeth of nearly equal size and shape were cleaned and stored in distilled water with $0.1 \%$ thymol crystal.

Specimen preparation: Each tooth was embedded in a cylindrical plastic mold that was filled with dental stone up to tooth cementoenamel junction.

Starting from mesio-incisal angle coordinate marking $5 \mathrm{~mm}$ gingivally and $5 \mathrm{~mm}$ distally along the incisal edge were joined together to form an imaginary triangle base with the apex corresponding to mesio-incisal line angle. A standardized experimental mesio-incisal angle fracture was done using hammer and chisel.

Specimen restoration Specimens were randomly divided into 2 groups thirty teeth each according to the type of composite resin used. Group I restored with Filtek Z350XT (3M ESPE, USA) and group II restored with Ceram X duo (Dentsply, Germany). Each group was further divided equally into three subgroups $\mathrm{A}, \mathrm{B}$ and $\mathrm{C}(\mathrm{N}=10)$ according to finish line preparation. A: no mechanical preparation, B: Circumferential shoulder (extended $2 \mathrm{~mm}$ cervically with a depth $0.5 \mathrm{~mm})$ with bevel $(1 \mathrm{~mm})$ preparation and C: Circumferential chamfer preparation (extended $2 \mathrm{~mm}$ cervically with a depth $0.5 \mathrm{~mm}$ ). (Figure 1 and 2).

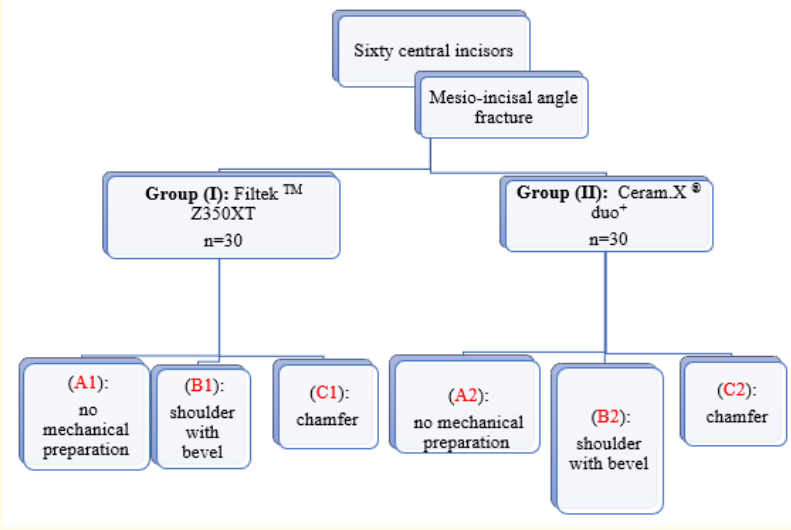

Figure 1: Schematic diagram of groups classification. 


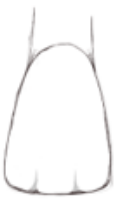

Sound tooth

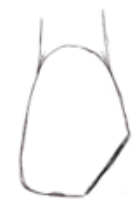

No mechanical preparation

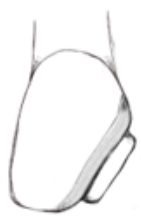

Shoulder with bevel

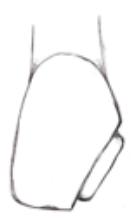

Chamfer
Figure 2: Different types of preparation techniques used in the study.

Teeth were restored with composite resin after shade selection, thermocycle between $5^{\circ} \mathrm{c}$ and $55^{\circ} \mathrm{c}$ for 500 cycles with a dwell time of 1 minute in each thermal bath and then they were photographed under standard illumination and same distances. Esthetic evaluation was performed by Photoshop computer programmer using CIE Lab system. Marginal adaptation was evaluated by stereomicroscope and scanning electron microscope after tooth sectioning into two halves mesio-incisal angle.

\section{Statistical analysis}

The collected data was tabulated and statistically analyzed using Graph Pad statistical software for Windows. Two-way ANOVA, One-way ANOVA and Student t-test were used to detect significance between preparation and composite. Chi square test was conducted for marginal adaptation analysis. $\mathrm{P}$ values $\leq 0.05$ are statistically significant in all tests.

\section{Results}

Regarding color match: There was no significant difference between subgroups in group I $(\mathrm{P}=0.5554)$ and in group II $(\mathrm{P}=$ 0.9298). No significant difference observed between subgroups $A$ $(\mathrm{P}=0.9438), \mathrm{B}(\mathrm{P}=0.5573)$ and $\mathrm{C}(\mathrm{P}=0.9465)$ in both groups (Table1, Figure 3).

Regarding marginal adaptation: No significant difference was observed between subgroups in group I ( $\mathrm{P}=1)$ and between subgroups of group II $(\mathrm{P}=0.7174)$. There was no significant difference between subgroups $A(P=0.716), B(P=0.707)$ and $C(P$ $=0.7165$ ) in both groups (Table 2, Figure 4-6).

\begin{tabular}{|l|c|c|c|}
\hline \multirow{2}{*}{$\begin{array}{l}\text { Group } \\
\text { Finish line } \\
\text { design }\end{array}$} & $\begin{array}{c}\text { Group I } \\
\text { (Z 350 XT) }\end{array}$ & $\begin{array}{c}\text { Group II } \\
\text { (Ceram X duo) }\end{array}$ & t-test \\
\cline { 2 - 4 } & Mean \pm SD & Mean \pm SD & P value \\
\hline Subgroup A & $2.3 \pm 0.42$ & $2.32 \pm 0.52$ & 0.9438 \\
\hline Subgroup B & $2.02 \pm 0.48$ & $2.2 \pm 0.62$ & 0.5573 \\
\hline Subgroup C & $2.23 \pm 0.53$ & $2.25 \pm 0.58$ & 0.9465 \\
\hline ANOVA (P value) & 0.5554 & 0.9298 & \\
\hline
\end{tabular}

Table 1: Color change for both composite groups regarding finish line design.
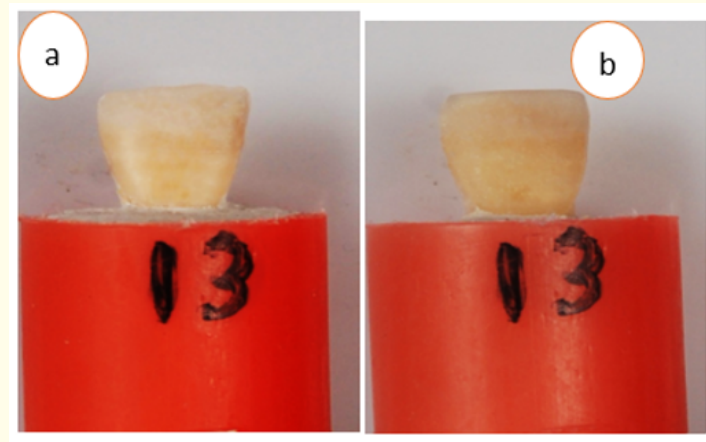

Figure 3: Sound tooth (a) (b) subgroup A after restoration (Z350XT) and thermocycling.
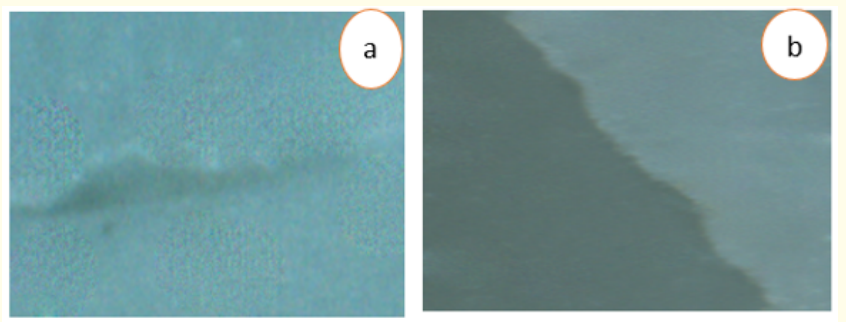

Figure 4: (a)Tooth in group 1 Shoulder with bevel showing gab formation (b) Tooth in group 1 Shoulder with bevel showing good adaptation. 

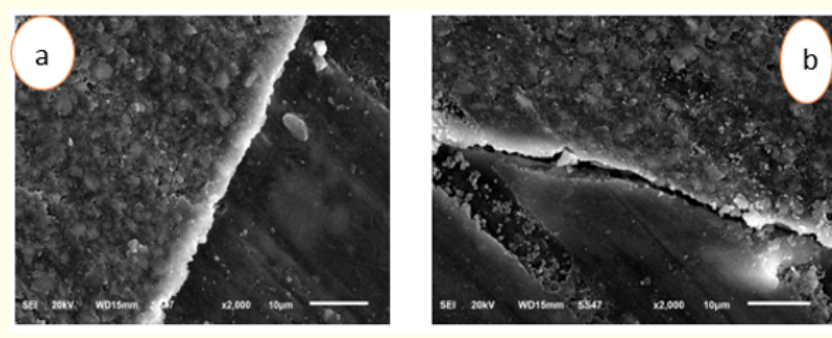

Figure 5: (a)Tooth in group II, chamfer preparation with good adaptation (b) Tooth in group II with chamfer with showing gap formation.
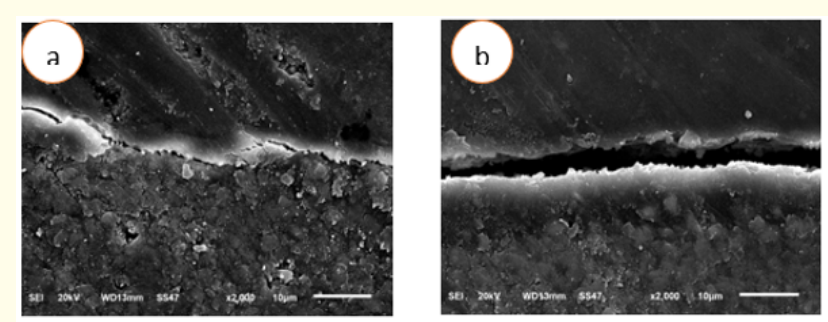

Figure 6: (a) Tooth in group II, shoulder with bevel preparation showing a slight gap (b) Tooth in group II, shoulder with bevel preparation showing gap formation.

\begin{tabular}{|c|c|c|c|c|c|c|c|}
\hline \multirow{3}{*}{\begin{tabular}{|l|} 
Group \\
Finish \\
line design \\
\end{tabular}} & \multicolumn{6}{|c|}{ Composite type } & \multirow{3}{*}{$\begin{array}{l}\text { Chi test } \\
\text { P value }\end{array}$} \\
\hline & \multicolumn{3}{|c|}{ Group I (Z 350 XT) } & \multicolumn{3}{|c|}{ Group II (Ceram X duo) } & \\
\hline & No gap & Gap at CSM & Gap at (E or D) & No gap & Gap at CSM & Gap at (E or D) & \\
\hline Subgroup A & $7(70 \%)$ & $1(10 \%)$ & $2(20 \%)$ & $7(70 \%)$ & $2(20 \%)$ & $1(10 \%)$ & 0.716 \\
\hline Subgroup B & $7(70 \%)$ & $1(10 \%)$ & $2(20 \%)$ & $6(60 \%)$ & $1(10 \%)$ & $3(30 \%)$ & 0.707 \\
\hline Subgroup C & $7(70 \%)$ & $1(10 \%)$ & $2(20 \%)$ & $7(70 \%)$ & $2(20 \%)$ & $1(10 \%)$ & 0.7165 \\
\hline Chi test & $P$ value & & 1 & $P$ value & & 7174 & \\
\hline
\end{tabular}

Table 2: Gap distribution in all studied subgroups.

\section{Discussion}

Dental trauma with anterior tooth fracture is a tragic experience for patients [26]. Different clinical protocols have been developed to fulfill the patient desire to have an esthetic appearance and the dentist to provide conservative restoration. One of the common conservative treatments is the direct adhesive restorations $[27,28]$.

In this study two nano-composite material were used Z350 $\mathrm{XT}$ and Ceram X duo. Z350 XT has unique nanoparticles (non agglomerated and non aggregated particles of $20 \mathrm{~nm}$ size) and nanoclusters ( nano-sized particles which are loosely agglomerated and act as a single unit) so increase filler loading and gives high strength. Ceram $\mathrm{X}$ duo is organically modified ceramic nanoparticles and nanofillers combined with conventional glass fillers of 1 micron, so it merges hybrid composite filler technology with advanced nano-technology [29].

Esthetic appearance was assessed through examination of restoration color match with remaining tooth structure. Evaluation of color match with naked eyes may lead to inaccuracy and inefficiency therefore, color match was evaluated using photographs and image analysis with Photoshop computer programme. Wee., et al. [30] recommended color evaluation with photographs as it presented high stability, efficiency and reliability, also Paris., et al. [31] suggested digital imaging for color difference evaluation.

Image analysis using computer programmer has the advantage of providing reproducible way for quantification of tooth color, transforming to a set of numerical values in terms of the CIELab system $\left(\mathrm{L}^{*}, \mathrm{a}^{*}, \mathrm{~b}^{*}\right)$ with the aid of photoshop computer programme. CIE Lab system can recognize small color difference, sensitive, and universally accepted [32,33].

In this study, color difference $(\Delta \mathrm{E})$ in both composite groups was less than 3.3 which is considered esthetically acceptable. This result is in a line with Mourouzis., et al. [34] who found no statistically significant difference among different composite types used (microhyprid, microfilled and nanofilled) and all provides acceptable color difference as $(\Delta \mathrm{E})$ was less than 3.3 . 
The results of our study showed no significant difference in $(\Delta \mathrm{E})$ between two types of composite in all three types of cavosurface margin preparations. This study agreed with Davis [35] who highly recommended the use of nanocomposite for restoring fractured anterior teeth. Nanocomposite have improved optical property due to very small filler size that is smaller than visible light wave length $(0.4-0.8 \mathrm{Mm})$, so particles cannot be detected by human's eye.

Also our result agreed with Terry [36], who stated that nanocomposite systems offered natural and stable interface between tooth and restoration, as tooth structure is nanoscopic $(1 \mathrm{~nm}-10 \mathrm{~nm})$ and filler particles are nanoscopic so this provides improvement in the continuity between them and natural appearance.

Also in this study, there was no significant difference between three types of preparation as all provide esthetic restorations. This agreed with Gondo [37] who concluded that there was no esthetic difference between different cavosurface configuration (no preparation and bevel) and all provides esthetic restorations and agreed with Strain [38] who found that shoulder with bevel cavosurface margin preparation provided esthetic restoration and masked finish line. Also this study is in a line with Jordan., et al. [39] who stated that chamfer preparation provides esthetic restoration with limited tooth preparation.

This result disagrees with Cai., et al. [40] who reported that, bevel preparation may decrease the color difference between the tooth and restoration. This may be due to difference in the methodology as they used premolars, and the cavity was prepared on buccal surface of the premolar instead of proxmo-incisal fracture and used different composite materials.

Results showed adequate marginal adaptation of fractured anterior teeth restored with either Z350 XT or Ceram X duo with different cavosurface margin preparations. The main goal of tooth restoration is to regain its form and function. If tight seal is not provided by the restoration, marginal gaps will be formed and will be colonized with micro-organisms that produce toxins that affects the pulp. Therefore, marginal integrity of restoration is a fundamental requirement as it affects restoration longevity [28].
This result was in a line with Hegde., et al. [41] who found no significant difference between Filtek Z350 XT and Ceram X duo. Nano-composite materials have very small filler particles (nanofiller) with increased the filler level and decreased the resin matrix. Since resin matrix is the main cause of polymerization shrinkage so its decrease leads to reduction of polymerization shrinkage and improvement in nanocomposite physical properties.

Also, in this study there was no significant difference between three types of cavo-surface margin regarding the marginal adaptation criteria. This was in a line with Davis., et al. [42] who found no significant difference in marginal integrity among different types cavo-surface margin preparation of microfill composite and agreed with Barrantes [43] who concluded that cavosurface margin configuration had no effect on clinical performance of direct composite resin restoration in fractured anterior teeth.

\section{Conclusions}

Based on the results of the present study we concluded that, both Nano-composite types and all three types of fracture margin preparations provided acceptable color match and esthetic restorations with well adapted margin.

\section{Conflict of Interests}

All authors deny any conflict of interest

\section{Author contributions}

Dr El- Kalla and Dr Shalan designed the study, drafted the initial manuscript, reviewed the search strategy and approved the final manuscript as submitted. Dr Mai, collected data, analyzed data, and approved the final manuscript as submitted.

\section{Funding statement}

Not financially supported.

\section{Acknowledgements}

The authors would like to thank everyone who help us in this work.

\section{Bibliography}

1. Diangelis A., et al. "International Association of Dental Traumatology guidelines for the management of traumatic dental injuries: 1. Fractures and luxations of permanent teeth". Jouranl dental traumatology 28.1 (2012): 2-12.

2. Andreasen J., et al. "Textbook and color atlas of traumatic injuries to the teeth, 4th edn". Oxford, UK: Wiley-Blackwell (2007). 
3. Traebert J., et al. "Prevalence, treatment needs, and predisposing factors for traumatic injuries to permanent dentition in 11-13 yearold schoolchildren". Cadernos de Saúde Pública 20.2 (2004): 403-410.

4. Hamilton F., et al. "An investigation of dentoalveolar trauma and its treatment in an adolescent population. Part 1: the prevalence and incidence of injuries and the extent and adequacy of treatment received". British Dental Journal 182.3 (1997): 91-95.

5. Andreasen J and Andreasen F. "Textbook and color atlas of traumatic injuries to the teeth". 3rd edn Copenhagen: Munsksgaard (1994).

6. Manju M., et al. "Esthetic and biologic mode of reattaching incisor fracture fragment utilizing glass fiber post2". Journal of Natural Science, Biology and Medicine 6.2 (2015): 446-448.

7. Chosack A and Eildeman E. "Rehabilitation of fractured incisor using the patient's natural crown. Case report". Journal of Dentistry for Children 31 (1964): 19-21.

8. Abdulkhayum A., et al. "In-vitro Evaluation of Fracture Strength Recovery of Reattached Anterior Fractured Tooth Fragment Using Different Re-Attachment Techniques". Journal of Clinical and Diagnostic Research 8.3 (2014): 208-211.

9. Kumar S., et al. "Multidisciplinary Approach in Management of Fractured Central Incisor through Composite Plug Stabilization - A Case Report". Journal of International Oral Health 5.1 (2013): 79-82.

10. PrabhakarA., et al. "Acomparison of impact strength of fragement-bonded anterior teeth using three different restorative materials: an in vitro study". Journal of Indian Society of Pedodontics and Preventive Dentistry 25.2 (2007): 88-92.

11. Nayak U., et al. "Restoration of fractured anterior permanent teeth using reference guide technique". BMJ Case Reports (2013).

12. Kulkarni V., et al. "Autogenous tooth fragment reattachment: A multidisciplinary management for complicated crown-root fracture with biologic width violation". Journal of Indian Society of Pedodontics and Preventive Dentistry 32.2 (2014):190194.
13. Roeters J. "Extended indications for directly bonded composite restorations: a clinicians view". The Journal of Adhesive Dentistry 3 (2001): 81-87.

14. Gandhi K and Nandlal B. "Effect of enamel preparations on fracture resistance of composite resin buildup of fractures involving dentine in anterior bovine teeth: an in vitro study". Journal of Indian Society of Pedodontics and Preventive Dentistry 24.2 (2006): 69-75.

15. Buonocore M and Davila J. "Restoration of fractured anterior teeth with ultraviolet-light-polymerized bonding materials: a new technique". The Journal of the American Dental Association 86.6 (1973):1349-1354.

16. Araujo J. "Influence of the cavosuperficial angle configuration in the esthetic result of direct restorations on fractured anterior teeth".189 f. Thesis (Doctor degree in dentistry) - postgraduate student, Federal university of santa catarina, Florianopolis (2003).

17. Gordon V. "In vitro evaluation of margins of replaced resinbased composite restoration". Journal of Esthetic and Restorative Dentistry 12.4 (2000): 209-215.

18. Milnar F. "Mastering minimal intervention and discretionary aesthetic procedures when placing direct composites". Practical Procedures and Aesthetic Dentistry 17.6 (2005): 428-432.

19. Bagheri J and Denehy G. "Effect of restoration thickness at the cavosurface bevel on the class IV acid-etched retained composite resin restoration". Journal of Prosthetic Dentistry 54.2 (1985): 175-177.

20. Felippe L., et al. "Clinical strategies for success in proximoincisal composite restorations. Part II: Composite application technique". Journal of Esthetic and Restorative Dentistry 17.1 (2005): 11-21.

21. Vargas M. "Conservative aesthetic enhancement of the anterior dentition using a predictable direct resin protocol". Practical Procedures and Aesthetic Dentistry 18.8 (2007): 501-507.

22. Davis M., et al. "Marginal integrity of adhesive fracture restoration: Chamfer Versus Bevel”. Quintessence International 11 (1983):1135-1139.

23. Strain R. "Class IV Composite Repair". Journal of Cosmetic Dentistry 30.1 (2014): 14-21. 
24. Okubo S., et al. "Evaluation of visual and instrument shade matching". Journal of Prosthetic Dentistry 80 (1998): 642-648.

25. Porte A., et al. "Cavity designs for composite resins". Operative Dentistry 9 (1984): 50-56.

26. Shahjad P., et al. "Reattach before it loses the natural integrity- Management of an anterior fractured teeth by fragment reattachment Baba Farid University". Journal of Dentistry 4.3 (2013): 164 -168.

27. Wieczkowski G., et al. "Microleakage in various dentine bonding agent/composite resin systems". Operative Dentistry 5 (1992): 62-67.

28. Terry D. "Direct composite resin restoration of adolescent class IV tooth fracture: a case report". Practical Periodontics and Aesthetic Dentistry 12.1 (2000): 23-29.

29. Singh S., et al. "Microleakage evaluation of nano ceramic and nano composite resins using self etch adhesive in class $\mathrm{V}$ cavities using single increment technique". Daryl Jackson Alastair Swayn 1.11 (2013): 106-111.

30. Wee A., et al. "Color accuracy of commercial digital cameras for use in dentistry". Dental Materials 22 (2006): 553-559.

31. Paris S., et al. "Masking of white spot lesions by resin infiltration in vitro". Journal of Dentistry 41 (2013): 28-34.

32. Lee B., et al. "Development of in vitro tooth staining model and usage of catalysts to elevate the effectiveness of tooth bleaching". Dental Materials 24 (2008): 57-66.

33. Ali s., et al. "Evaluation of the Bleaching Efficacy and Dentin Microhardness after Using Thiourea Combined with Hydrogen Peroxide for NonVital Bleaching of Discolored Teeth. Thesis (M.S.)- Mansoura university. Faculty of Dentistry". Department of conservative Dentistry (2010).

34. Mourouzis P., et al. "Color match of resin composites to intact tooth structure". Journal of Applied Biomaterials and Functional Materials 13.3 (2015): e259-e265.

35. Davis N. "A nanotechnology composite". Compendium of Continuing Education in Dentistry 24 (2003): 662-670.

36. Terry D. "Direct applications of a nanocomposite resin system: Part 1-The evolution of contemporary composite materials". Practical Procedures and Aesthetic Dentistry 16.6 (2004): 417422.
37. Gondo R. Influence of cavo-surface configuration in the esthetic result of composite resin restoration in anterior fractured teeth - An invitro study 155 (2003).

38. Strain R. "Class IV Composite Repair". Journal of Cosmetic Dentistry 30.1 (2014): 14-21.

39. Jordan R., et al. "Restoration of fractured and hypoplastic incisors by the acid etch resin technique: a three-year report". The Journal of the American Dental Association 95 (1977): 795-803.

40. Cai X., et al. "Effects of different cavosurface margins on color matching of the resin composite". Journal of Peking University 47.1 (2015):120-123.

41. Hegde M., et al. "A comparative evaluation of microleakage of three different newer direct composite resins using a selfetching primer in class V cavities: An in vitro study". Journal of Conservative Dentistry 12.4 (2009):160-163.

42. Davis M., et al. "Marginal integrity of adhesive fracture restoration: Chamfer Versus Bevel". Quintessence International 11 (1983): 1135-1139.

43. Barrantes J., et al. "Clinical Evaluation of Direct Composite Resin Restorations in Fractured Anterior Teeth". International Journal of Dental Sciences 16 (2014): 47-61.

\section{Volume 3 Issue 11 November 2019} (C) All rights are reserved by Hanaa Mahmoud Shalan., et al. 